\title{
Über Heparin und Erfindungen in der Medizin im Allgemeinen
}

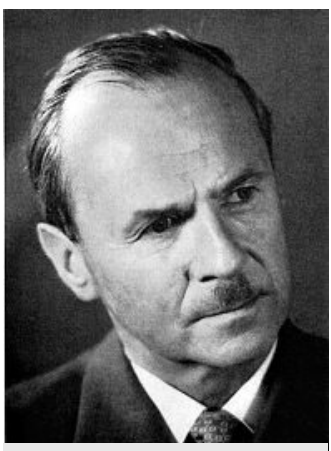

Karl Lenggenhager (1903-1989) steht für die Schweizer Wurzeln der Heparin-Prophylaxe.

Herzlichen Dank an meinen Kollegen Thomas Schlich (McGill University, Montreal).

* Die Literaturangaben finden sich in der Online-Version unter www.saez.ch $\rightarrow$ Aktuelle Nummer oder $\rightarrow$ Archiv $\rightarrow 2011 \rightarrow 28 / 29$.

** PD Dr. rer. soc. Eberhard Wolff ist Kulturwissenschaftler, Medizinhistoriker und Mitglied der Redaktion Medizingeschichte der Schweizerischen Ärztezeitung.
Ein kleiner Fehltritt im Dunklen hatte für mich vor ein paar Jahren ungeahnte Wirkungen. Nicht nur der gebrochene Mittelfussknochen und der in der Notfallstation erhaltene Gips waren ziemlich neue Erfahrungen, sondern auch, dass ich gleichzeitig für einige Zeit unter die «Selbstspritzer» gehen musste: subkutane Thromboseprophylaxe.

Völlig normal, wird sich der Praktiker denken. Auch die postoperative Thromboseprophylaxe, sagt Urs Lenggenhager, ist heute eine völlige Selbstverständlichkeit. Sie zu unterlassen käme einem Kunstfehler gleich. Weniger bekannt, meint der Berner Chirurg, seien die soliden Schweizer Wurzeln der Heparin-Prophylaxe: Sein Vater Karl Lenggenhager (1903-1989) erforschte seit den frühen 30er-Jahren am Inselspital die Geheimnisse der Blutplättchen und der Blutgerinnung, publizierte eifrig darüber und führte als Klinikchef an der Insel 1941 den sogenannten «5er Stoss» als generelle postoperative Thromboseprophylaxe mit bemerkenswertem Erfolg ein. Das war vor 70 Jahren und ist heute Grund genug, die SÄZ-Redaktion daran zu erinnern.

«Wer hat's erfunden?»- ein Schweizer, wie in der Ricola-Werbung? Eine kleine Online-Recherche über die Einführung des Heparins führt zu reichlich disparaten Ergebnissen. Ein schweizerisches Lexikon sagt, Lenggenhager habe die Prophylaxe mit niedrig dosiertem Heparin eingeführt [1]. Der ehemalige Berner Angiologe Felix Mahler meint 1996, Lenggenhager habe diese Praxis «wahrscheinlich» experimentell begründet und erwähnt daneben den Heparineinsatz des Stockholmer Chirurgen Clarence Crafoord [2]. Eine deutsche Quelle betont die Gleichzeitigkeit der ersten klinischen Anwendungen bei beiden Chirurgen im Jahr 1935 [3]. Zwei schwedische Autoren wiederum stellen Crafoords klinische Studien in den Mittelpunkt und erwähnen weitere parallele Pionierstudien in Toronto [4]. Eine kanadische Quelle, wen wundert's, erwähnt nur die Letzteren durch den dortigen Chirurgen Gordon Murray [5, 6]. Bereits 1960 liess der Hamburger «Spiegel» seine Leser wissen, welche Klinik das Heparin als «erstes Krankenhaus der Welt» ab 1948 regelmässig eingesetzt habe. Wir ahnen es schon: Es sei die Universitäts-Frauenklinik in Hamburg gewesen [7].

Wer was erfunden haben soll, ist oft eine Frage der Perspektive. Die Medizingeschichte kennt ein ähnliches, berühmteres Beispiel hierfür. Googeln wir die Wortfolge «Begründer der Bakteriologie» auf Deutsch, finden wir sie doppelt so oft mit dem Namen «Koch» assoziiert wie mit dem Namen «Pas- teur». Geben wir stattdessen «fondateur de la bactériologie» in die Suchmaske ein, ist das Zahlenverhältnis beinahe umgekehrt.

Nach ein paar Monaten intensiver historischer Forscherarbeit liessen sich wahrscheinlich einige der Fragen zur Schweizer Rolle bei der Einführung der Heparinprophylaxe genauer beantworten. Ich vermute, dass, wie so oft, engagiert forschende Kliniker an vielen Orten mehr oder weniger gleichzeitig an diesem Thema gearbeitet haben, nicht immer ohne Konkurrenzgedanken.

«Wer hat's erfunden?» Für die Medizingeschichte stellt sich die Frage weniger als für die findigen Ricola-Werbestrategen, weil medizinische Entwicklungen meist komplexe Prozesse sind, die nicht auf ein einzelnes «Heureka!» oder «Ich wag's!» reduziert werden können. Aber das macht es ja gerade so spannend! Auch den klinischen Erstanwendern fiel die Heparinprophylaxe nicht aus dem Himmel in den OP. Erst musste das Heparin chemisch isoliert werden (das war bereits 1916). Die antikoagulante Wirkung musste entdeckt werden. Dann musste es in grösseren Mengen in nicht toxischer, reiner und bezahlbarer Form gewonnen werden können [8]. Selbst die klinische Erstanwendung bedeutete noch keine Durchsetzung in der Medical Community und keine Implementation in der alltäglichen Praxis. Diese sei nach grossen Studien erst in den 1970er Jahren gekommen [3].

Auch Lenggenhagers Sohn weist auf die langsame Implementation hin, obwohl sein Vater (und ebenso die anderen damit beschäftigten Kollegen, E. W.) ihre Ergebnisse vielfältig publizierten. Der Sohn erklärt es damit, dass sein Vater «kein Mensch der lauten Publizistik» war. Mahler vermutet in der deutschen Publikationssprache einen Grund oder in dem Umstand, dass das Publikationsorgan Helvetica Medica Acta 1941 (!) in einem deutschen Verlag (Thieme, Leipzig) erschien [3].

Solche Fragen sind Leckerbissen für die Science Studies, die mit ihrem methodischen Repertoire systematisch versuchen, Antworten aus der Spekulation in die Plausibilität zu überführen. Der Berner Karl Lenggenhager zählte sicher zu den Pionieren bei der Einführung der Heparinprophylaxe. Sein Berner Engagement wäre vielleicht ein Lehrstück für die Komplexität von Erfindungen in der Medizingeschichte.

Eberhard Wolff **

PS: Das gilt im Übrigen genauso für die Kräuterzucker! 


\section{Literatur}

1 www.hls.ch

2 Mahler F. Aus der Geschichte der Thromboseverhütung und -behandlung. Therapeutische Umschau. 1996;53:254-7.

3 Kujath P. Die ambulante Thromboseprophylaxe. Deutsches Aerzteblatt. 1995;92:A2003-6. www.aerzteblatt.de/pdf/92/28/a2003.pdf

4 Escobar Kvitting JP, Olin CL. Clarence Crafoord. A Giant in Cardiothoracic Surgery, the First to Repair Aortic Coarctation. Ann Thorac Surg. 2009;87:342-6. http://ats.ctsnetjournals.org/cgi/content/ full $/ 87 / 1 / 342$
5 Rutty C. Miracle Blood Lubricant. Connaught and the Story of Heparin, 1928-1937. Contact. 1996;9. www.healthheritageresearch.com/Heparin-Conntact9608.html

6 Marcum JA. The Development of Heparin in Toronto. J Hist Med Allied Sci. 1997;52:310-37. Marcum erwähnt nebenbei auch die Stockholmer Versuche, nicht aber die Berner.

7 Dünnes Blut. Der Spiegel vom 27.7.1960. www.spiegel.de/spiegel/print/d-43067605.html

8 Marcum JA. The Origin of the Dispute over the Discovery of Heparin. J Hist Med Allied Sci. 2000;55:37-66. http://muse.jhu.edu/journals/jhm/ summary/v055/55.1marcum.html 